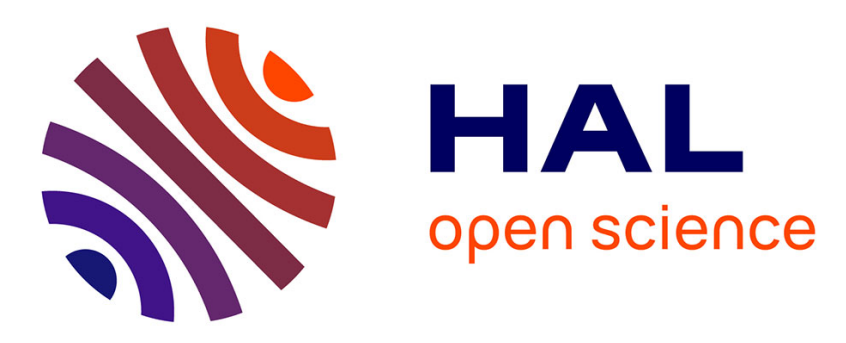

\title{
Nanobeam X-ray Fluorescence Dopant Mapping Reveals Dynamics of in Situ Zn-Doping in Nanowires
}

\author{
Andrea Troian, Gaute Otnes, Xulu Zeng, Lert Chayanun, Vilgaile Dagyte, \\ Susanna Hammarberg, Damien Salomon, Rainer Timm, Anders Mikkelsen, \\ Magnus T. Borgstrom, et al.
}

\section{To cite this version:}

Andrea Troian, Gaute Otnes, Xulu Zeng, Lert Chayanun, Vilgaile Dagyte, et al.. Nanobeam X-ray Fluorescence Dopant Mapping Reveals Dynamics of in Situ Zn-Doping in Nanowires. Nano Letters, 2018, 18 (10), pp.6461-6468. 10.1021/acs.nanolett.8b02957 . hal-02976305

\section{HAL Id: hal-02976305 https://hal.science/hal-02976305}

Submitted on 23 Oct 2020

HAL is a multi-disciplinary open access archive for the deposit and dissemination of scientific research documents, whether they are published or not. The documents may come from teaching and research institutions in France or abroad, or from public or private research centers.
L'archive ouverte pluridisciplinaire HAL, est destinée au dépôt et à la diffusion de documents scientifiques de niveau recherche, publiés ou non, émanant des établissements d'enseignement et de recherche français ou étrangers, des laboratoires publics ou privés. 


\title{
Nanobeam X-ray Fluorescence Dopant Mapping Reveals Dynamics of in Situ Zn-Doping in Nanowires
}

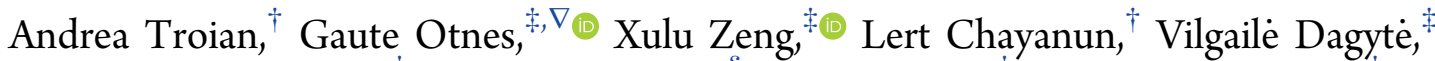 \\ Susanna Hammarberg, ${ }^{\dagger}$ Damien Salomon, ${ }^{\S}$ Rainer Timm, ${ }^{\dagger}$ Anders Mikkelsen, \\ Magnus T. Borgström, ${ }^{\ddagger 0}$ and Jesper Wallentin* ${ }^{\dagger \dagger}$ \\ ${ }^{\dagger}$ Synchrotron Radiation Research and NanoLund, Lund University, 22100 Lund, Sweden \\ ${ }^{\ddagger}$ Solid State Physics and NanoLund, Lund University, 22100 Lund, Sweden \\ ${ }^{\S}$ European Synchrotron Radiation Facility, 38000 Grenoble, France
}

\section{Supporting Information}

ABSTRACT: The properties of semiconductors can be controlled using doping, making it essential for electronic and optoelectronic devices. However, with shrinking device sizes it becomes increasingly difficult to quantify doping with sufficient sensitivity and spatial resolution. Here, we demonstrate how $\mathrm{X}$-ray fluorescence mapping with a nanofocused beam, nano-XRF, can quantify $\mathrm{Zn}$ doping within in situ doped III-V nanowires, by using large area detectors and high-efficiency focusing optics. The spatial resolution is defined by the focus size to $50 \mathrm{~nm}$. The detection limit of $7 \mathrm{ppm}\left(2.8 \times 10^{17} \mathrm{~cm}^{-3}\right)$, corresponding to about $150 \mathrm{Zn}$ atoms in the probed volume, is bound by a background signal. In solar cell InP nanowires with a $\mathrm{p}$-i-n doping profile, we use nano-XRF to observe an

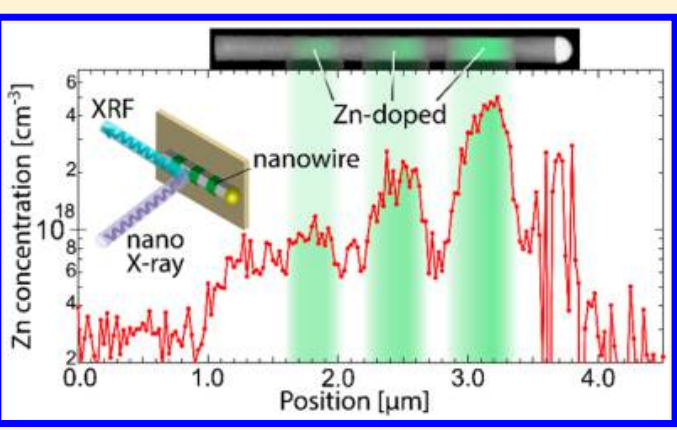
unintentional $\mathrm{Zn}$ doping of $5 \times 10^{17} \mathrm{~cm}^{-3}$ in the middle segment. We investigated the dynamics of in situ $\mathrm{Zn}$ doping in a dedicated multisegment nanowire, revealing significantly sharper gradients after turning the $\mathrm{Zn}$ source off than after turning the source on. Nano-XRF could be used for quantitative mapping of a wide range of dopants in many types of nanostructures.

KEYWORDS: Nanowire, III-V, nano-XRF, doping, InGaP, InP

C ontrol of intentional and unintentional impurity doping is essential for the performance of electronic and optoelectronic devices. Doping of nanoscale devices poses many additional challenges compared with bulk devices, such as donor deactivation ${ }^{1}$ and anisotropic incorporation, ${ }^{2}$ and even the position of single dopants can alter the properties of modern field-effect transistors. ${ }^{3}$ Precise characterization methods are crucial to control and understand impurity doping, but nanostructures pose a challenge for traditional bulk tools. The spatial resolution needs to be on the order of $10 \mathrm{~nm}$, which should preferably be maintained in $3 \mathrm{D}$, with as low detection limit as possible.

The two main established methods for doping characterization are Hall effect measurements ${ }^{4}$ and secondary ion mass spectrometry (SIMS). ${ }^{5}$ Both offer low detection limits for thin films, ${ }^{6}$ but the in-plane spatial resolution is limited. Optical techniques such as photoluminescence and Raman spectroscopy can quantify carrier concentration in nanostructures, ${ }^{7-9}$ but the spatial resolution is constrained by the diffraction limit. Other options available for nanostructures ${ }^{10}$ are atom probe tomography (APT) and electron-beam induced energy dispersive X-ray spectroscopy (EDS). Although pulsed laser $\mathrm{APT}^{11,12}$ has demonstrated $\mathrm{ppm}$ detection limits and subnanometer resolution, it requires complex sample prepara- tion and is restricted to specific sample geometries, and similarly to SIMS it is a destructive technique. EDS is readily available in electron microscopes with high spatial resolution, but the detection limit is normally above $0.1 \%$ (1000 ppm).

$\mathrm{X}$-ray fluorescence (XRF) exhibits a much lower detection limit than EDS for elements with atomic numbers above $20 .^{13}$ Similarly to EDS, an elemental map can be created by scanning the sample in a focused beam, in a nondestructive process. Although the spatial resolution of $\mathrm{X}$-ray methods has traditionally been quite poor, X-ray optics have developed quickly in recent years. The spatial resolution at established synchrotron nanofocusing beamlines is typically about 100 $\mathrm{nm},{ }^{14}$ and even sub-10 nm focusing has been demonstrated. $^{15,16}$

Early studies used XRF with a nanofocused beam, nano$\mathrm{XRF}$, to investigate single $\mathrm{ZnO}$ nanowires that were homogeneously doped with ion implantation of Co to a concentration of $0.3 \%$ (3000 ppm). ${ }^{17}$ Here, we employ highend X-ray optics and XRF detectors to demonstrate a spatial resolution of $50 \mathrm{~nm}$ and a detection limit of $7 \mathrm{ppm}$ in the case

Received: July 20, 2018

Revised: $\quad$ August 27, 2018

Published: September 5, 2018 
of $\mathrm{Zn}$-doped InP nanowires. The high sensitivity, almost 3 orders of magnitude better than previously reported, paired with the excellent spatial resolution, establishes nano-XRF as a promising tool for the characterization of nanoscale electronics and optoelectronics.

We employed nano-XRF to investigate III-V nanowires that were in situ doped with $\mathrm{Zn}$ during growth. Semiconductor nanowires are promising candidates for novel electronics and optoelectronics applications ${ }^{18}$ due to the possibility of designing complex heterostructures by assembling different materials and crystal types in the same nanostructure. ${ }^{19,20}$ Impressive performance has been demonstrated in a wide range of devices ${ }^{21-24}$ and materials, ${ }^{25-27}$ and in situ doping of nanowires during growth has been performed using a variety of different dopants. ${ }^{0}$

$\mathrm{Zn}$ is a common p-dopant for III-V materials, but due to the lack of ohmic electrical contacts, quantitative measurement of p-doping in InP nanowires has remained very challenging. The lack of proper characterization tools has in turn hampered studies of in situ $\mathrm{Zn}$ incorporation in nanowires. Investigations of doped thin films have shown that $\mathrm{Zn}$ can exhibit both memory effects and solid diffusion, ${ }^{28}$ but there is little known about in situ $\mathrm{Zn}$ doping of nanowires. Using nano-XRF, we quantitatively show that in situ $\mathrm{Zn}$ doping of nanowires does exhibit memory effects. We investigate the switching dynamics and find a strong asymmetry, with significantly sharper gradients after turning the source off than after turning the source on.

We first present a comparison of nano-XRD and EDS for a $\mathrm{Zn}$-doped $\mathrm{Ga}_{x} \mathrm{In}_{1-x} \mathrm{P}$ nanowire. An undoped $\mathrm{InP}$ reference nanowire was subsequently used to establish the detection limit. We then measured a solar cell InP nanowire with an axial p-i-n doping profile, in order to investigate the spatial resolution and to explore the doping in the nominally undoped middle section. Finally, we investigated the incorporation of $\mathrm{Zn}$ when switching on and off the dopant source, using InP nanowires with alternating nominally undoped and $\mathrm{p}$ doped segments. We report data for only one nanowire per sample in the main text, but for each sample the consistency of the results was confirmed by measuring several nanowires (as reported in S1 the Supporting Information).

All nanowires were grown via Metal Organic Vapor Phase Epitaxy (MOVPE) from Au seed particles arranged in an array by nanoimprint lithography, ${ }^{29}$ resulting in large controlled arrays of nominally identical nanowires. In situ $\mathrm{Zn}$-doping was performed by introducing the precursor diethyl zinc (DEZn) during growth. The morphology of the nanowires was assessed by scanning electron microscopy (SEM) after growth (Figure 1a). The as-grown nanowires were broken off the substrates and deposited on $\mathrm{Si}_{3} \mathrm{~N}_{4}$ X-ray transparent windows for the nano-XRF measurements (Figure $1 b$ ).

The hard X-ray beam available at the ID16B beamline at the ESRF synchrotron facility ${ }^{30}$ was used as a probe. The multilayer-coated Kirkpatrick-Baez focusing mirrors of this beamline create a $50 \mathrm{~nm}$ focus in so-called pink beam mode, which gives a very high flux by using a moderately monochromatic beam $\left(\Delta E / E \approx 10^{-2}\right)$. In this mode, the $\mathrm{X}$ ray energy cannot be tuned. To capture as much as possible of the XRF signal, six large area silicon drift detectors were placed close to the sample.

We first compare the nano-XRF and EDS methods for measuring the dopant concentration in $\mathrm{Zn}$-doped $\mathrm{Ga}_{x} \mathrm{In}_{1-x} \mathrm{P}$ nanowires (Figure $2 \mathrm{a}$ ). The Ga concentration was independ-

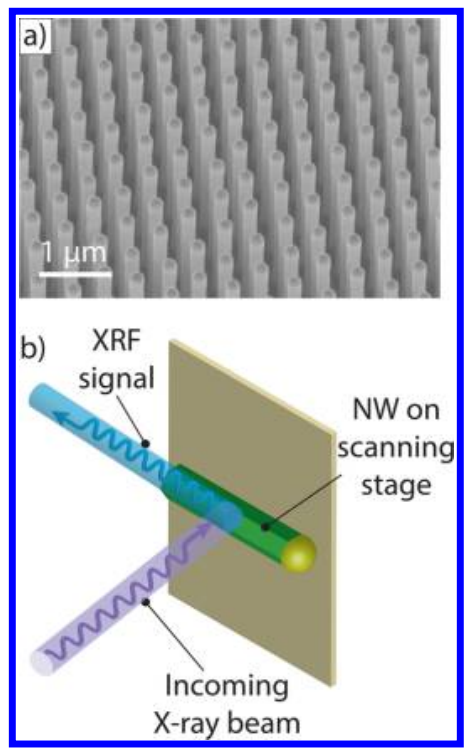

Figure 1. Samples and experimental overview. (a) SEM image of nanowires as grown on the substrate. (b) Schematics of the experiment. The nanowires are deposited on a $\mathrm{Si}_{3} \mathrm{~N}_{4}$ window and scanned under a nanofocused X-ray beam, while the XRF signal at each point is detected.

ently characterized by X-ray diffraction (XRD) measurements (average $x=0.35$ ). The spatial distributions of $\mathrm{Au}, \mathrm{In}, \mathrm{Zn}$, and Ga mapped by nano-XRF are represented in an image with each primary color channel corresponding to an element (Figure 2b). We also performed one-dimensional line scans using EDS and nano-XRF (Figure 2d,e), as well as point measurements with longer counting times.

The comparison between two point spectra taken in the middle of the nanowire (Figure 2c) shows the lower background of the nano-XRF compared to that for EDS. The background signal in EDS is due to bremsstrahlung, as the electrons in the beam scatter with the electrons in the sample. In contrast, X-rays are absorbed in a one-step process that lacks the bremsstrahlung component, and this makes it possible to detect minute concentrations of $\mathrm{Zn}$. The concentration of $\mathrm{Zn}$ detected in the Au particle with EDS is an artifact, which has to be attributed to the vicinity of the $\mathrm{Au} L$ line with the $\mathrm{Zn} \mathrm{K}$ line in the XRF spectrum. This implies that $\mathrm{Zn}$ cannot be estimated in a matrix of Au. The P K line is weaker in nano-XRF than in EDS due to the air absorption. We used the known atomic concentration of $\mathrm{Ga}$ as a quantitative reference for the $\mathrm{Zn}$ concentration (as explained in detail in the Methods section). Due to the higher background, the EDS line scan (Figure 2d) exhibits much more noise than the XRF line scan (Figure 2e).

Both nano-XRF and EDS show a gradient in the Ga and In concentrations along the nanowire axis, with an increasing $\mathrm{Ga}$ concentration toward the top. The opposite trend was found for the In concentration, preserving the total fraction of the group III elements as expected. The In is supplied primarily via surface diffusion, and we assume that the In concentration therefore reduces with increasing distance from the substrate. ${ }^{31}$ The top part of the nanowire near the $\mathrm{Au}$ seed, i.e., the last segment to grow, was observed to be In-rich due to precipitation from the Au-alloy particle during cool down. Compositional gradients in ternary nanowires have been previously observed by many groups, ${ }^{32-35}$ since these gradients can be readily measured by EDS. 
a)

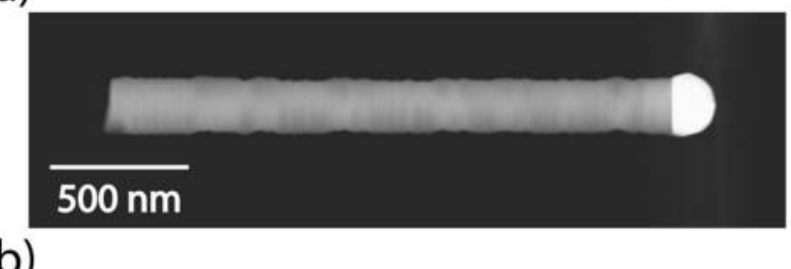

b)

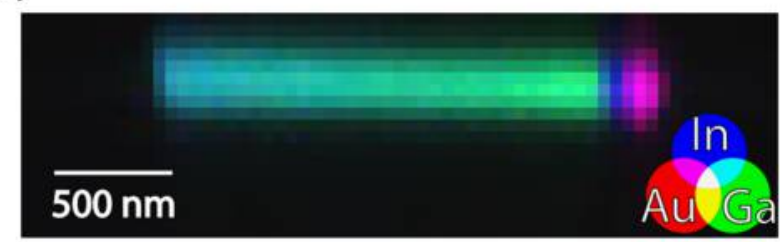

C)

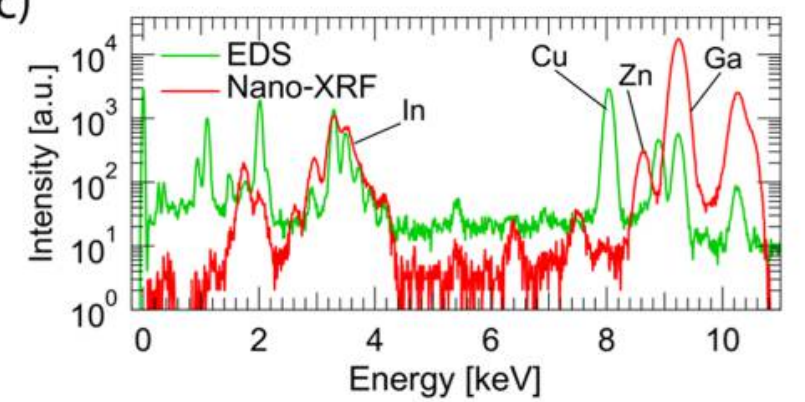

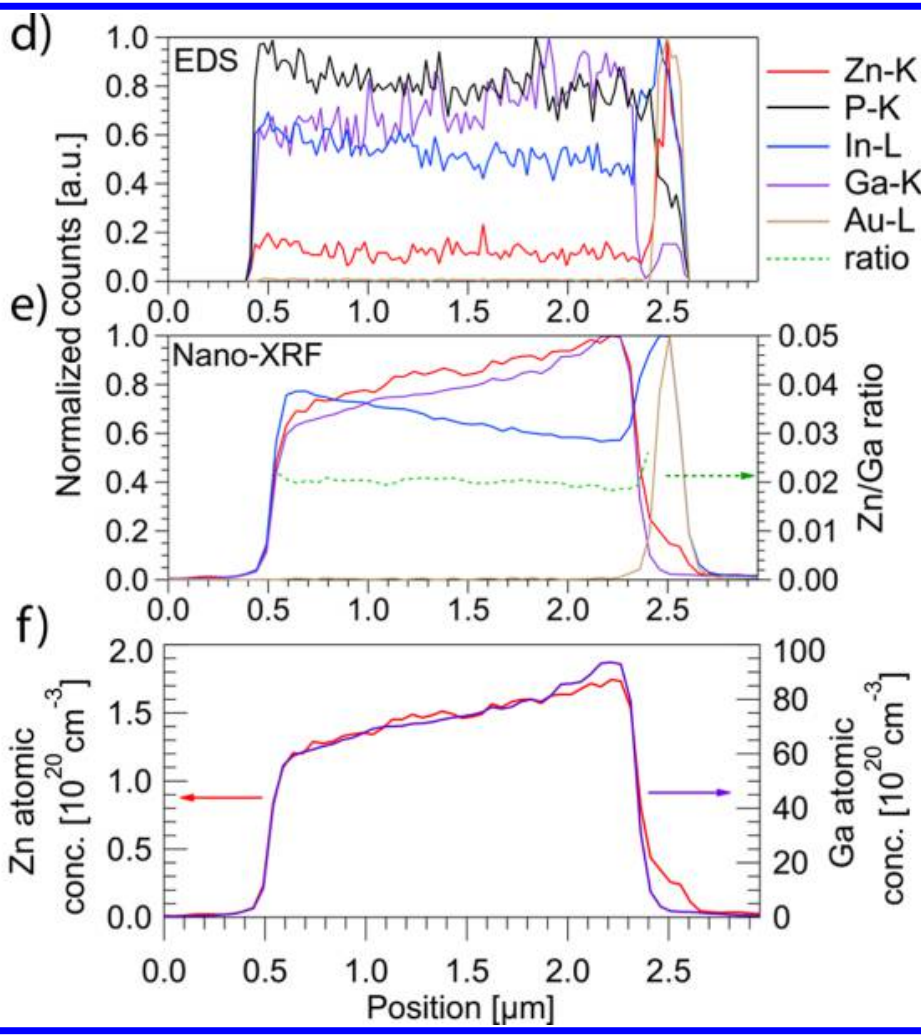

Figure 2. Zn-doping quantification by nano-XRF. (a) STEM image of a $\mathrm{Ga}_{x} \mathrm{In}_{1-x} \mathrm{P}$ nanowire (not the same nanowire as for nano-XRF, but from the same sample). (b) Nano-XRF color map of a Zn-doped $\mathrm{Ga}_{x} \mathrm{In}_{1-x} \mathrm{P}$ nanowire ( $0.5 \mathrm{~s}$ per pixel, $50 \mathrm{~nm}$ step size). (c) EDS and XRF spectra from points in the nanowires in (a) and (b). (d) Normalized EDS counts from a line scan along the nanowire in (a) (200 s per point, $50 \mathrm{~nm}$ step size). (e) Normalized nano-XRF line scans along the nanowire in (b) (5 s per point, $50 \mathrm{~nm}$ step size). (f) Atomic concentrations of $\mathrm{Zn}$ and Ga in the nanowire in (b).

Previous EDS-based investigations of $\mathrm{Ga}_{x} \mathrm{In}_{1-x} \mathrm{P}$ nanowires by our group have shown that DEZn increases the Ga fraction in the nanowires, ${ }^{36}$ but that study was unable to determine the $\mathrm{Zn}$ concentration since it was below the detection limit of the EDS system. In contrast, we are here able to quantify the $\mathrm{Zn}$ concentration using nano-XRF, exploiting the signal of the $\mathrm{Zn}$ $\mathrm{K}$ line $(8.6 \mathrm{keV})$, which is clearly distinguishable from the $\mathrm{Ga}$ $\mathrm{K}$ line $(9.2 \mathrm{keV})$. The $\mathrm{Zn}$ concentration is perfectly correlated with the $\mathrm{Ga}$ concentration, at a constant ratio of 0.018 (Figure $2 \mathrm{e})$. The atomic concentration of $\mathrm{Zn}$ increases from $c=1.2 \times$ $10^{20} \mathrm{~cm}^{-3}$ at the base to $c=1.7 \times 10^{20} \mathrm{~cm}^{-3}$ at the top (Figure $2 \mathrm{f}$ ). The reason for the $\mathrm{Zn}-\mathrm{Ga}$ correlation is unclear, but we note that $\mathrm{Zn}$ has an atomic radius similar to that for $\mathrm{Ga}$. $\mathrm{Zn}$ may preferentially incorporate on Ga sites, which could lead to the observed correlation.

The detection limits of nano-XRF are a crucial point of the discussion. In the pioneering work of Co doping evaluation in $\mathrm{ZnO}$ nanowires mentioned before, ${ }^{17}$ the detection limits were assessed using a certified standard, ${ }^{37}$ with results similar to ours. We investigated the detection limit using a blank sample (i.e., an undoped NW) instead. This choice was led by the lack of a certified standard having a geometry and material and matrix effects similar to those of our samples. Considering the broad variety in definitions and methods for detection and quantification limits, ${ }^{38,39}$ we here clarify the procedure with which those quantities were assessed for $\mathrm{Zn}$ doping evaluation with nano-XRF. We defined the detection limit $\left(L_{\mathrm{D}}\right)$ according to the International Union of Pure and Applied Chemistry (IUPAC) recommendations. ${ }^{40}$ Assuming a normal distribution of the measurements of the blank (Figure 3a), a simplified

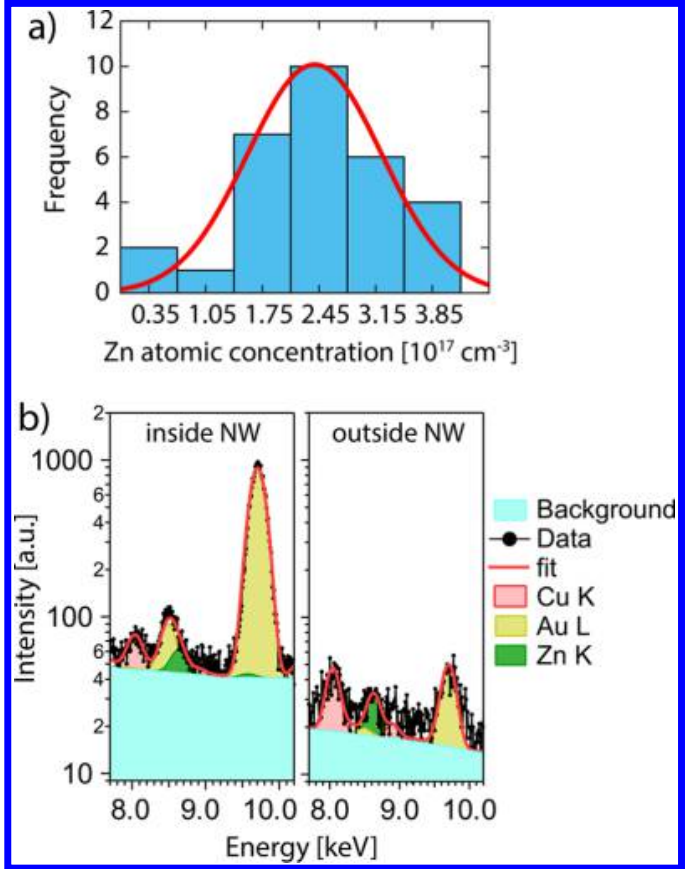

Figure 3. Determination of the detection limit with an undoped InP nanowire. (a) Histogram with the Gaussian distribution fit (red curve) of 30 individual XRF measurements on the blank, an undoped InP nanowire. The average is $\mu_{\mathrm{B}}=2.4 \times 10^{17} \mathrm{~cm}^{-3}$, and the standard deviation is $\sigma_{\mathrm{B}}=8 \times 10^{16} \mathrm{~cm}^{-3}$. (b) XRF spectra acquired with $100 \mathrm{~s}$ counting time in the $\mathrm{Zn} \mathrm{K}$ edge region inside and outside of the nanowire. 
relation for the estimation of the concentration is provided by $L_{\mathrm{D}}=3.4 \sigma_{\mathrm{B}}$, where $\sigma_{\mathrm{B}}$ is the standard deviation of the blank (details can be found in Supporting Information S2). One of the most recommended approaches, which has been used here, consists of statistical hypothesis testing of the blank sample, excluding errors of first (false positives) and second types (false negatives).

We evaluated $L_{\mathrm{D}}$ by using a nominally undoped InP nanowire as blank. The reactor was cleaned in an acid bath and dry etched with $\mathrm{HCl}$ before the nanowire growth, in order to avoid any contamination from previous growth runs with DEZn. We acquired 30 XRF measurements of $10 \mathrm{~s}$ each on the blank sample (Figure 3a,b). The blank showed an average $\mathrm{Zn}$ concentration of $\mu_{\mathrm{B}}=2.4 \times 10^{17} \mathrm{~cm}^{-3}$, with a standard deviation of $\sigma_{\mathrm{B}}=8 \times 10^{16} \mathrm{~cm}^{-3}$ (Figure 3a). We therefore estimate our detection limit to $L_{\mathrm{D}}=3.4 \sigma_{\mathrm{B}}=2.8 \times 10^{17} \mathrm{~cm}^{-3}$ (i.e., $7 \mathrm{ppm}$ ), for $10 \mathrm{~s}$ measurements. Such a concentration is equivalent to about $150 \mathrm{Zn}$ atoms in the probed volume at a given point. The estimate of $L_{\mathrm{D}}$ scales with the standard deviation of the blank signal, which could possibly be reduced with longer counting times. However, substantial beam damage and sample drift were observed with very long counting times, and $10 \mathrm{~s}$ was found to be a good compromise between a good signal quality and those shortcomings.

The average signal for the blank is similar to the signal measured several micrometers away from the blank nanowire $\left(1.6 \times 10^{17} \mathrm{~cm}^{-3}\right)$. We therefore argue that this background signal stems from the experimental environment, for instance due to $\mathrm{Zn}$ used as an alloy element in $\mathrm{Al}$ parts typically used in the experimental equipment. Although this average signal is a background for our measurements, which could in principle be subtracted from the following results, we present the following data with background for clarity.

Due to the lack of high-resolution characterization tools, it has previously not been possible to study the dynamics of in situ $\mathrm{Zn}$ doping in nanowires. Therefore, after having established a quantitative scale for the $\mathrm{Zn}$ nano-XRF signal and having determined the detection limit, we applied this method on solar cell InP nanowires with an axial p-i-n doping profile (Figure 4). An ideal p-i-n diode should have as low doping as possible in the middle segment, but $\mathrm{Zn}$ is prone to various types of memory effects from previously grown segments. $^{28,41}$ Since $\mathrm{Zn}$ is a metal that is expected to be present in high concentrations in the seed particle, it could create a reservoir effect. ${ }^{42}$ In addition, $\mathrm{Zn}$ and its precursors could have carry over effects originating from species adsorbed on the sample surface and MOVPE reactor walls. ${ }^{43}$

The line scan (Figure 4) along the nanowire axis clearly shows the p-doped bottom segment, followed by a sharp decay to the middle segment. The sharp axial doping profile demonstrates the spatial resolution with the $50 \mathrm{~nm}$ focus. We measured a $\mathrm{Zn}$ concentration of $c=3 \times 10^{19} \mathrm{~cm}^{-3}$ in the center of the p-doped segment (Figure 4a). A 2D map of the same nanowire is available in the section S3 of the Supporting Information. Additionally, XRF spectra were taken with 10 repeated measurements of $10 \mathrm{~s}$ count times each, at specific points of interest along the nanowire; in Figure 4b, we show a point outside the nanowire (A), in the p-doped segment (B), and in the nominally undoped segment $(\mathrm{C})$. The data (Figure 4a) also suggest that $\mathrm{Zn}$ is present in the $\mathrm{Au}$ seed, but a quantification was not possible in this point since the $\mathrm{Zn} \mathrm{K}$ line and $\mathrm{Au} \mathrm{L}$ lines are energetically close and the peak area ratio of $\mathrm{Au} \mathrm{L}$ to $\mathrm{Zn} \mathrm{K}$ is about 120.

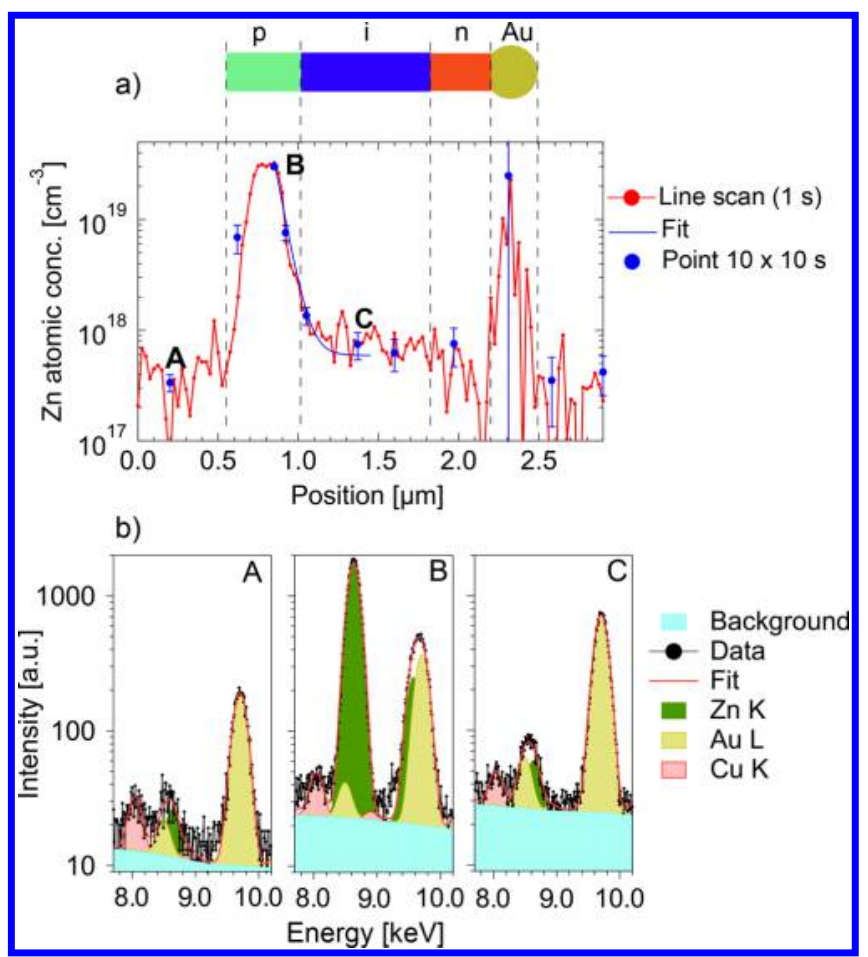

Figure 4. Nano-XRF of a $\mathrm{p}-\mathrm{i}-\mathrm{n}$ InP nanowire revealing background doping in the nominally undoped middle segment. (a) Atomic concentration of $\mathrm{Zn}$ along the $\mathrm{InP}$ p-i-n nanowire (1 s per point, 25 $\mathrm{nm}$ step), which is shown with its nominal doping profile schematically at the top. The blue dots show the average of point measurements of 10 times $10 \mathrm{~s}$ each. The error bars represent their standard deviation. The blue line shows a fit of the trailing edge of the p-segment obtained with eq. 1. (b) XRF spectra of $100 \mathrm{~s}$ point measurements in the $\mathrm{Zn} \mathrm{K}$ edge region recorded at positions $\mathrm{A}$ (outside), B (p-segment), and C (nominally undoped segment).

We measured an average $\mathrm{Zn}$ concentration $c=(8 \pm 2) \times$ $10^{17} \mathrm{~cm}^{-3}$ along the nominally undoped middle segment. The $\mathrm{Zn}$ signal acquired a few microns away from the nanowire was $c=(3 \pm 1) \times 10^{17} \mathrm{~cm}^{-3}$, which is comparable with the background measured in the undoped blank nanowire, $\mu_{\mathrm{B}}=2.4$ $\times 10^{17} \mathrm{~cm}^{-3}$. The concentration in the middle segment was therefore estimated to $c=5 \times 10^{17} \mathrm{~cm}^{-3}$, by subtracting the background. This is clearly higher than the detection limit $L_{\mathrm{D}}=$ $2.8 \times 10^{17} \mathrm{~cm}^{-3}$. Note also that the $10 \mathrm{~s}$ point measurements are consistent with the faster line scans ( $1 \mathrm{~s}$ for each point). The drift was typically $30 \mathrm{~nm} / \mathrm{h}$ or less, which leads to a negligible loss of resolution in single line scans that were acquired within less than $10 \mathrm{~min}$. The standard deviation (about $1.2 \times 10^{17} \mathrm{~cm}^{-3}$ ) of the point measurements taken on the sharp decay of the p-segment can be attributed to the drift of the sample between the subsequent $10 \mathrm{~s}$ measurements and it is still below the detection limit. The unintentional $\mathrm{Zn}$ concentration in the middle segment could be the result of memory effects in the reactor or a reservoir effect in to the seed particle, as discussed further below. The high unintentional $\mathrm{Zn}$ doping in the middle segment means that the depletion region of the p-i-n junction is shorter than intended, which could result in a poor solar cell efficiency.

The nano-XRF method opens up for studies of the dynamics of switching during in situ doping, but the single junction in the $\mathrm{p}-\mathrm{i}-\mathrm{n}$ doped sample gives limited insight. In order to obtain more information on the dynamics of $\mathrm{Zn}$ incorporation when switching the DEZn dopant source on and off, we therefore 
prepared dedicated InP nanowires with multiple junctions in a barcode doping profile. These nanowires had four nominally undoped segments (denoted as I0, I1, I2, and I3 in Figure 5)

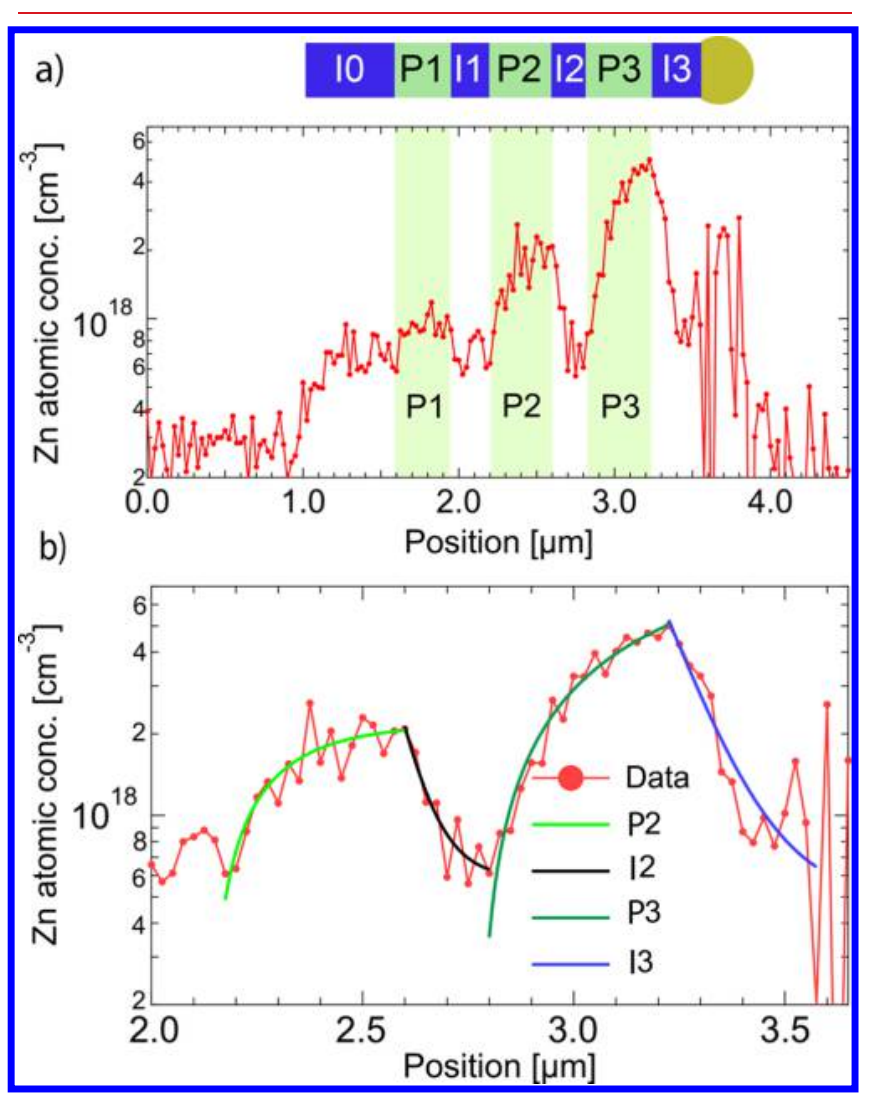

Figure 5. Nano-XRF revealing asymmetric $\mathrm{Zn}$-doping profiles in a barcode i-p-i InP nanowire. (a) Measured $\mathrm{Zn}$ concentration in a barcode i-p-i-p-i-p-i InP nanowire (3 s per point, $25 \mathrm{~nm}$ steps). The structure is shown schematically above the measured doping profile. Three p-segments with increasing doping are shown (highlighted in green and denominated as P1, P2, P3) with nominally undoped segments between (denominated as I0, I1, I2, I3). (b) Measured concentration profiles of $\mathrm{P} 2, \mathrm{I} 2, \mathrm{P} 3$, and $\mathrm{I} 3$, together with fits using eq. 1.

and three Zn-doped segments of increasing DEZn molar fraction (P1, P2, and P3). Each segment was approximately $300 \mathrm{~nm}$ long as revealed by in situ monitoring by optical reflectometry, ${ }^{44}$ significantly longer than the $50 \mathrm{~nm}$ beam diameter.

With nano-XRF the P2 and P3 segments with $\mathrm{Zn}$ concentrations of $c=2 \times 10^{18} \mathrm{~cm}^{-3}$ and $c=5 \times 10^{18} \mathrm{~cm}^{-3}$, respectively, could be clearly distinguished from the nominally undoped regions (Figure 5a). The lowest-doped segment P1 is just barely identifiable, with an average count rate of only $25 \%$ above the I0 and I1 segments. It is worth noting that a plateau is visible in the $\mathrm{Zn}$ doping profile for segment P2, whereas this is not the case for segment P3 (Figure 5b). The background $\mathrm{Zn}$ concentration, measured away from the nanowire, was on average $c=(2.8 \pm 0.6) \times 10^{17} \mathrm{~cm}^{-3}$, similar to the background for the undoped reference nanowire and the $\mathrm{p}-\mathrm{i}-\mathrm{n}$ nanowire.

Even though the DEZn source was switched instantaneously, the line scans clearly show gradual increases and decreases of the $\mathrm{Zn}$ concentration. One possible reason for the slow dynamics could be that $\mathrm{Zn}$ is stored in the Au seed particle and is gradually built up and released as the source is switched. We developed a model based on this reservoir effect, similar to an approach by $\mathrm{Li}$ and Gösele ${ }^{42}$ (derivation in the section S4 of the Supporting Information). The $\mathrm{Zn}$ concentrations $c$ as a function of the axial position from the edge, $x$, at the leading and trailing edges of the p-segments, were fitted with

$$
c(x)=c_{1}+\left(c_{2}-c_{1}\right) \mathrm{e}^{-x / 1}
$$

Here $c_{1}$ is the initial dopant concentration in the nanowire when the gas source is switched on/off, $c_{2}$ is the steady state concentration at an infinite distance, and $l$ is a characteristic length. During the fitting $c_{1}, c_{2}$, and $l$ were set as free parameters. Equation 1 can be used to fit both the transient behavior in the leading edges (i-p junctions, after the DEZn source is switched on) and the trailing edges ( $p-i$ junctions, after the DEZn source is switched off) of the $\mathrm{Zn}$ concentration.

The fitting results are summarized in Table 1 . The characteristic lengths of the trailing edges were $l=0.06 \pm$ $0.02 \mu \mathrm{m}$ for $\mathrm{I} 2$ and $l=0.10 \pm 0.02 \mu \mathrm{m}$ for I3. Since the characteristic lengths are similar to the $50 \mathrm{~nm}$ beam size, we cannot claim with certainty that the differences are significant. The $\mathrm{p}$-i junction in the $\mathrm{p}-\mathrm{i}-\mathrm{n} \mathrm{InP}$ nanowire showed a similar characteristic length: $l=0.056 \pm 0.004 \mu \mathrm{m}$.

Assuming the reservoir effect as the cause for the transient behavior, $l$ should be constant and the same for both the leading and the trailing edges of the doping profile in the barcode nanowire. In contrast, we find that the characteristic lengths of the leading edges were significantly longer than the trailing edges, i.e., $l=0.7 \pm 0.4 \mu \mathrm{m}$ for P3 and $l=0.16 \pm 0.09$ $\mu \mathrm{m}$ for P2. The length of the P3 segment $(0.3 \mu \mathrm{m})$ was not sufficient to allow the $\mathrm{Zn}$ concentration to reach its steady state value $c_{2}=1.1 \times 10^{19} \mathrm{~cm}^{-3}$. The results of the fittings for the barcode nanowire suggest that higher doping levels require longer segment lengths to reach the steady state concentration. The ratio of the steady state concentrations $c_{2}$ in P3 and P2 is about 5 , not 10 as expected from the ratio of the DEZn molar

Table 1. Growth and Fitting Parameters of the $\operatorname{In}_{x} \mathrm{Ga}_{1-x} \mathbf{P}$ Barcode $\mathrm{NW}^{a}$

$\begin{array}{ccccc}\text { segment } & \text { molar fraction } \chi_{\text {DEZn }} & \text { growth rate }[\mathrm{nm} / \mathrm{s}] & \text { characteristic length, } l_{\text {on } / \text { off }}[\mu \mathrm{m}] & \text { steady state concentration, } c_{1}\left[\mathrm{~cm}^{-3}\right] \quad \text { initial concentration, } c_{2}\left[\mathrm{~cm}^{-3}\right] \\ \text { I0 } & 0 & 2.37 & 2.21 & \\ \text { P1 } & 1.1 \times 10^{-7} & 1.97 & 0.16(0.09) & 2.2(0.3) \times 10^{18} \\ \text { I1 } & 0 & 1.63 & 0.06(0.02) & 6(2) \times 10^{17} \\ \text { P2 } & 1.1 \times 10^{-6} & 1.44 & 0.7(0.4) & 1.1(0.5) \times 10^{19} \\ \text { I2 } & 0 & 1.17 & 0.010(0.02) & 6(3) \times 10^{17} \\ \text { P3 } & 1.1 \times 10^{-5} & 1.07 & 5(2) \times 10^{17}\end{array}$

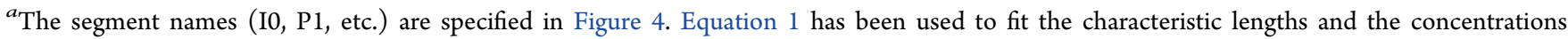
showed above. 
Table 2. Growth Parameters for All the Sets of Nanowires Investigated in This Work ${ }^{a}$

\begin{tabular}{|c|c|c|c|c|c|c|c|c|}
\hline \multirow[b]{2}{*}{ nanowire batch } & \multirow[b]{2}{*}{ segment } & \multicolumn{6}{|c|}{ molar fractions } & \multirow[b]{2}{*}{ segment length $(\mu \mathrm{m})$} \\
\hline & & $\chi_{\mathrm{TMGa}}$ & $\chi_{\text {TMIn }}$ & $\chi_{\mathrm{PH}_{3}}$ & $\chi_{\mathrm{HCl}}$ & $\chi_{\mathrm{DEZn}}$ & $\chi_{\mathrm{TESn}}$ & \\
\hline $\mathrm{Ga}_{x} \mathrm{In}_{1-x} \mathrm{P}$ nanowires & $\mathrm{p}$ & $4.0 \times 10^{-4}$ & $5.2 \times 10^{-5}$ & $6.9 \times 10^{-3}$ & $5.4 \times 10^{-5}$ & & & 2 \\
\hline \multirow[t]{3}{*}{$\mathrm{p}$-i-n InP nanowires } & $\mathrm{p}$ & & $5.9 \times 10^{-5}$ & $6.9 \times 10^{-3}$ & $4.6 \times 10^{-5}$ & $4.6 \times 10^{-5}$ & & 0.35 \\
\hline & $\mathrm{i}$ & & $7.4 \times 10^{-5}$ & $6.9 \times 10^{-3}$ & $4.6 \times 10^{-5}$ & & & 1 \\
\hline & $\mathrm{n}$ & & $5.9 \times 10^{-5}$ & $6.9 \times 10^{-3}$ & $4.6 \times 10^{-5}$ & & $4.3 \times 10^{-5}$ & 0.07 \\
\hline \multirow[t]{7}{*}{ barcode InP nanowires } & I0 & & $7.4 \times 10^{-5}$ & $6.9 \times 10^{-3}$ & $4.6 \times 10^{-5}$ & & & 0.6 \\
\hline & $\mathrm{P} 1$ & & $7.4 \times 10^{-5}$ & $6.9 \times 10^{-3}$ & $4.6 \times 10^{-5}$ & $1.1 \times 10^{-7}$ & & 0.3 \\
\hline & I1 & & $7.4 \times 10^{-5}$ & $6.9 \times 10^{-3}$ & $4.6 \times 10^{-5}$ & & & 0.3 \\
\hline & $\mathrm{P} 2$ & & $7.4 \times 10^{-5}$ & $6.9 \times 10^{-3}$ & $4.6 \times 10^{-5}$ & $1.1 \times 10^{-6}$ & & 0.3 \\
\hline & $\mathrm{I} 2$ & & $7.4 \times 10^{-5}$ & $6.9 \times 10^{-3}$ & $4.6 \times 10^{-5}$ & & & 0.3 \\
\hline & P3 & & $7.4 \times 10^{-5}$ & $6.9 \times 10^{-3}$ & $4.6 \times 10^{-5}$ & $1.1 \times 10^{-5}$ & & 0.3 \\
\hline & $\mathrm{I} 3$ & & $7.4 \times 10^{-5}$ & $6.9 \times 10^{-3}$ & $4.6 \times 10^{-5}$ & & & 0.3 \\
\hline nominally undoped nanowires & i & & $7.4 \times 10^{-5}$ & $6.9 \times 10^{-3}$ & $4.6 \times 10^{-5}$ & & & 2 \\
\hline
\end{tabular}

fractions. Thus, the $\mathrm{Zn}$ concentration is not proportional to the DEZn molar fraction, as would be expected.

To conclude the discussion about the barcode NW, all edges can be fitted with a model based on asymptotic increase and exponential decay, respectively, with single characteristic decay lengths for each junction. However, the switching dynamics are strongly asymmetric, with much sharper gradients after switching DEZn off than on. This shows that effects other than a simple reservoir model are governing the concentration after the source is switched on, but we can only speculate regarding the underlying reason. The asymmetry has practical implications for devices where sharp junctions are needed, such as tunnel diodes used in multijunction solar cells. ${ }^{45}$ Some dopants, such as Be in GaAs, are known to diffuse relatively long distances in the solid host, ${ }^{46}$ in addition to the mechanisms discussed above. The internal diffusion of $\mathrm{Zn}$ in the NW cannot be considered a relevant mechanism here, since it would have resulted in a symmetric gradient of $\mathrm{Zn}$ along the NW (Figure 5b), whereas we observed a sharp transition when the dopant source was switched off. Furthermore, the characteristic length when the dopant source is switched off is smaller; i.e., the gradient is sharper for the I3 segment compared to that for I2 (Table 1), whereas a diffusion mechanism would have instead had a larger driving force due to the higher concentration gradient. Instead, we speculate that after switching the source on, physisorbed $\mathrm{Zn}$ is accumulated on the sample surface and reactor walls. As the surface concentration of $\mathrm{Zn}$ increases and saturates, the flux of $\mathrm{Zn}$ into the seed particle slowly increases. However, after the $\mathrm{Zn}$ source is turned off, the $\mathrm{Zn}$ on the surface quickly evaporates.

Modern nanoscale devices require dopant characterization with very high spatial resolution and low detection limit. We have shown that nano-XRF is a powerful technique to address this issue, with a low detection limit and a high spatial resolution that make it possible to follow the dynamics of dopant switching within in situ doped nanowires. Nano-XRF can be applied for many types of nanostructures and nanodevices, using almost any combination of dopants and host material.

We find that the lowest detectable concentration in the case studied here, $\mathrm{Zn}$ doping of $\mathrm{III}-\mathrm{V}$ nanowires, is as low as 7 $\mathrm{ppm}$. Measurements on a reference sample indicate that it is limited by a background signal in the system, which suggests that it could be improved by careful design of the experiment.
Novel detectors with extremely large solid angles would increase the detected signal, ${ }^{47}$ as would the even higher X-ray fluxes from the new diffraction-limited synchrotrons that are being built. ${ }^{48}$ The detection limit will generally depend on the dopant element, since this determines the XRF energy. Ideally, the X-ray excitation energy should be tuned to just above the absorption edge of the element of interest. For lighter elements with lower XRF emission energies, soft X-ray excitation could enhance the sensitivity.

The spatial resolution shown here, $50 \mathrm{~nm}$, is still a bit too poor for modern integrated circuits, which have feature sizes of around $10 \mathrm{~nm}$. However, the spatial resolution of nano-XRF could be improved to below $10 \mathrm{~nm}$ using emerging X-ray optics. ${ }^{15,16}$ In addition, the 3D distribution of dopants would be revealed by tomography. In nanowires, for example, 3D measurements could detect radial elemental distribution gradients, and reveal whether dopants are preferentially incorporated at the surface. ${ }^{49}$ Tomography would put limitations on acquisition time per point, in order to overcome possible beam damage issues.

In addition to improving the sensitivity and spatial resolution, there are several open paths for developing nanoXRF for dopant studies. An open question in our measurements is whether the $\mathrm{Zn}$ atoms are incorporated correctly into the host lattice. By scanning the primary X-ray energy, an X-ray absorption near-edge structure (XANES) study can show how dopants are incorporated in the lattice, ${ }^{17}$ and differentiate between desirable substitutional and undesirable interstitial incorporation. Finally, we note that the long absorption length makes hard X-rays uniquely suited for in situ studies, which could allow studies of doping during crystal growth or ion implantation.

Methods. Nanowire Growth. Four distinct batches of $\mathrm{Ga}_{x} \operatorname{In}_{1-x} \mathrm{P}, \mathrm{p}$-i-n, barcode, and unintentionally doped InP nanowires were grown via metal organic vapor phase epitaxy (MOVPE) from Au seed particles patterned by nanoimprint lithography (as described elsewhere ${ }^{29}$ ) in a hexagonal pattern with $500 \mathrm{~nm}$ pitch on an InP:Zn (111)B substrate. The nanowires were grown with the growth parameters reported in Table 2. HCl was supplied in situ ${ }^{50,51}$ to prevent radial growth. The nanowire and its segment lengths were monitored in situ by optical reflectometry ${ }^{44}$ to $2 \mu \mathrm{m}$ for the $\mathrm{Ga}_{x} \mathrm{In}_{1-x} \mathrm{P}$ nanowires and to $\sim 0.35,1.00$, and $0.07 \mu \mathrm{m}$ for the $\mathrm{p}$-, $\mathrm{i}$-, and n-segments, respectively. The barcode $\mathrm{InP}$ nanowires exhibit a total of 
seven segments alternating between nominally intrinsic and increasingly $\mathrm{p}$-doped ones. Their length was also monitored by optical reflectometry, and the growth time was adjusted to yield segment lengths of $\sim 300 \mathrm{~nm}$, except for the first segment, which was grown to $600 \mathrm{~nm}$. The growth temperature was 440 ${ }^{\circ} \mathrm{C}$, and constant molar fractions of $\chi_{\mathrm{PH}_{3}}, \chi_{\mathrm{TMIn}}$, and $\chi_{\mathrm{HCl}}$ were supplied during the whole nanowire growth. SEM analysis showed that the diameter of $\mathrm{Ga}_{x} \mathrm{In}_{1-x} \mathrm{P}$ and $\mathrm{InP}$ nanowires measured $182 \pm 2 \mathrm{~nm}$ and $187 \pm 4 \mathrm{~nm}$, respectively.

TEM-EDS. A JEOL 3000F TEM operated at $300 \mathrm{kV}$, equipped with an energy dispersive X-ray spectroscopy (EDS) detector was used for imaging and chemical analysis. The nanowire image was taken in high-angle annular dark field scanning TEM (HAADF-STEM) mode. The elemental distribution and composition analysis were obtained from In $\mathrm{L} \alpha, \mathrm{Ga} \mathrm{K} \alpha, \mathrm{P} \mathrm{K} \alpha$, Au $\mathrm{L} \alpha, \mathrm{Zn} \mathrm{K} \alpha$ peaks, respectively. Standardless quantification was used. The step size was $10 \mathrm{~nm}$. In order to have a comparable step size with the step size of nano-XRF on $\mathrm{Ga}_{x} \mathrm{In}_{1-x} \mathrm{P}$ (i.e., $50 \mathrm{~nm}$ ), we binned the EDS data for five steps.

Nano-XRF. The $\mathrm{Ga}_{x} \mathrm{In}_{1-x} \mathrm{P}$ sample was first investigated using a monochromatic beam with an energy of $10.7 \mathrm{keV}$ and an energy resolution of $1 \mathrm{eV}$ focused on the sample with Kirkpatrick-Baez (KB) mirrors. The estimated beam size at the sample position was $65 \times 65 \mathrm{~nm}^{2}$, with an estimated flux of $9.36 \times 10^{10}$ photons/s. The InP and $\mathrm{Ga}_{x} \mathrm{In}_{1-x} \mathrm{P}$ samples were then measured with a pink beam $\left(\mathrm{d} E / E \approx 10^{-2}\right)$ at $17.5 \mathrm{keV}$ in order to take advantage of the higher flux $\left(7.3 \times 10^{11}\right.$ photons/ s) available at that energy, which is beneficial for the $\mathrm{Zn}$ evaluation in the nominally undoped segments. In this case, the beam spot size was $50 \times 60$ (horizontal $\times$ vertical) $\mathrm{nm}^{2}$.

The XRF signal was detected using two three-element Sidrift detectors oriented at $75^{\circ}$ to the sample normal. Highresolution $2 \mathrm{D}$ maps were acquired by scanning the nanowire under the nanofocused beam using piezoelectric motors and a step size of $25 \mathrm{~nm}$ ( $50 \mathrm{~nm}$ for the $\mathrm{Ga}_{x} \operatorname{In}_{1-x} \mathrm{P}$ ) and collecting the local XRF signal. In order to improve the statistical quality of the XRF line scans, multiple sweeps were carried out. The line scans were shifted in order to compensate for sample drift, using the $\mathrm{Au}$ seed particle as a reference. $2 \mathrm{D}$ maps were collected with an acquisition time of $0.5 \mathrm{~s}$ per pixel, whereas the line scans had an acquisition time from 1 to $5 \mathrm{~s}$ per point. Specific points of interest in the map were also selected and measured with long counting times.

XRF Analysis and Zn Quantification. The XRF spectra were fitted with the Pymca package. ${ }^{52}$ All relevant elements present in the sample and in the experimental environment were taken into account. Also, a STRIP background and pileup and escape peaks were considered to consider the properties of the detector electronics. We point out that due to the negligible thickness of the sample (about $180 \mathrm{~nm}$ ), the $\mathrm{X}$-ray scattering and absorption, and the consequent matrix effects, are weak (the primary X-ray absorption is $0.2 \%$ for $\operatorname{InP}$ NWs). We, however, considered them in the fitting. No shadowing effects are expected due to the fact that the NWs are not bent and are lying perfectly horizontal on the substrate. We mapped the spatial distribution of the relevant elements in the nanowires by considering the intensity of the $\mathrm{Ga} \mathrm{K} \alpha, \mathrm{Zn}$ $\mathrm{K} \alpha, \mathrm{Au} \mathrm{M} \alpha$, and $\mathrm{In} \mathrm{L} \alpha$ transition lines.

In order to assess quantitatively the local dopant concentration in the nanowires, we considered that the total atomic concentration in the $\mathrm{Ga}_{x} \mathrm{In}_{1-x} \mathrm{P}$ nanowire was $n_{\text {tot }}=4.3$ $\times 10^{22} \mathrm{~cm}^{-3}$. On the basis of the average atomic Ga fraction of $x=0.35$, obtained by $\mathrm{X}$-ray diffraction measurement, we calculated the $\mathrm{Ga}$ atomic concentration, $n_{\mathrm{Ga}}=7.5 \times 10^{21} \mathrm{~cm}^{-3}$. This number was used to define a conversion factor $k_{\mathrm{Ga}}$ scaling the average of the $\mathrm{Ga} K \alpha$ counts along the nanowire with the average $\mathrm{Ga}$ atomic concentration. We confirmed that the total atomic concentration of $\mathrm{Ga}$ and In was constant along the whole nanowire as expected (more details in the S5 and S6 sections of Supporting Information). A conversion factor $k_{\mathrm{Zn}}$ was defined to quantify the local $\mathrm{Zn} \mathrm{K} \alpha$ counts and to obtain the local $\mathrm{Zn}$ concentration. It was derived from $k_{\mathrm{Ga}}$ by taking into account the different absorption coefficients and XRF yields for the $\mathrm{Ga} \mathrm{K} \alpha$ and $\mathrm{Zn} \mathrm{K} \alpha$ lines. Also, the $\mathrm{X}$-ray flux, which was not directly measured, was considered. Because the $\mathrm{X}$-ray flux varied during the experiment, we introduced a scaling factor by calibrating the In $\mathrm{L} \alpha$ counts of the sample to those of the reference.

\section{ASSOCIATED CONTENT}

\section{Supporting Information}

The Supporting Information is available free of charge on the ACS Publications website at DOI: 10.1021/acs.nanolett.8b02957.

Consistency of the results, detection limit details, nanoXRF mapping of a $\mathrm{p}-\mathrm{i}-\mathrm{n}$ InP nanowire, reservoir model of the $\mathrm{Zn}$ doping, sum of $\mathrm{Ga}$ and In atomic concentrations, $\mathrm{Ga}_{x} \mathrm{In}_{1-x} \mathrm{P}$ nanowire measured at 17.5 $\mathrm{keV}$ (PDF)

\section{AUTHOR INFORMATION}

\section{Corresponding Author}

*Jesper Wallentin. E-mail: jesper.wallentin@sljus.lu.se. ORCID $\odot$

Gaute Otnes: 0000-0002-4241-5234

Xulu Zeng: 0000-0002-6269-2415

Rainer Timm: 0000-0001-8914-5924

Magnus T. Borgström: 0000-0001-8061-0746

Jesper Wallentin: 0000-0001-5909-0483

\section{Present Address}

${ }^{\nabla}$ G.O.: Institute for Energy Technology, 2007 Kjeller, Norway Author Contributions

A.T. performed the data analysis. G.O., X.Z. and M.T.B. grew the nanowires. A.T., L.C., V.D., S.H., D.S. and J.W. performed the measurements. R.T. and A.M. provided guidance through the project. A.T. and J.W. wrote the paper with support of all the other authors.

\section{Notes}

The authors declare no competing financial interest.

\section{ACKNOWLEDGMENTS}

We thank Erik Malm for help with measurements. We acknowledge the European Synchrotron Radiation Facility, Grenoble, France, for provision of synchrotron radiation facilities. Financial support by the Röntgen-Ångström Cluster, NanoLund, Marie Sklodowska Curie Actions, Cofund, Myfab, Swedish energy agency, Project INCA 600398, and the Swedish Research Council grant number 2015-00331 is gratefully acknowledged. The authors acknowledge the People Programme (Marie Curie Actions) of the European Union's Seventh Framework Programme (FP7-People-2013-ITN) under REA grant agreement no. 608153, PhD4Energy. 


\section{REFERENCES}

(1) Björk, M. T.; Schmid, H.; Knoch, J.; Riel, H.; Riess, W. Nat. Nanotechnol. 2009, 4, 103.

(2) Dastjerdi, M. H. T.; Fiordaliso, E. M.; Leshchenko, E. D.; Akhtari-Zavareh, A.; Kasama, T.; Aagesen, M.; Dubrovskii, V. G.; LaPierre, R. R. Nano Lett. 2017, 17 (10), 5875-5882.

(3) Koenraad, P. M.; Flatté, M. E. Nat. Mater. 2011, 10, 91.

(4) Storm, K.; Halvardsson, F.; Heurlin, M.; Lindgren, D.; Gustafsson, A.; Wu, P. M.; Monemar, B.; Samuelson, L. Nat. Nanotechnol. 2012, 7, 718.

(5) Chia, A. C. E.; Boulanger, J. P.; LaPierre, R. R. Nanotechnology 2013, 24 (4), 045701.

(6) Antell, G. R.; Briggs, A. T. R.; Butler, B. R.; Kitching, S. A.; Stagg, J. P.; Chew, A.; Sykes, D. E. Appl. Phys. Lett. 1988, 53 (9), $758-760$.

(7) Metaferia, W.; Sivakumar, S.; Persson, A. R.; Geijselaers, I.; Wallenberg, R. L.; Deppert, K.; Samuelson, L.; Magnusson, M. $\underline{H}$. Nanotechnology 2018, 29 (28), 285601.

(8) Ketterer, B.; Uccelli, E.; Fontcuberta i Morral, A. Nanoscale 2012, 4 (5), 1789-1793.

(9) Goktas, N. I.; Fiordaliso, E. M.; LaPierre, R. R. Nanotechnology 2018, 29 (23), 234001.

(10) Wallentin, J.; Borgström, M. T. L. Mater. Res. 2011, 26 (17), 2142-2156.

(11) Perea, D. E.; Lensch, J. L.; May, S. J.; Wessels, B. W.; Lauhon, L. I. Appl. Phvs. A: Mater. Sci. Process. 2006, 85 (3), 271-275.

(12) Perea, D. E.; Allen, J. E.; May, S. J.; Wessels, B. W.; Seidman, D. N.; Lauhon, L. I. Nano Lett. 2006, 6 (2), 181-185.

(13) Rackwitz, V.; Ostermann, M.; Panne, U.; Hodoroaba, V.-D. I. Anal. At. Spectrom. 2013, 28 (9), 1466-1474.

(14) Johansson, U.; Vogt, U.; Mikkelsen, A. NanoMAX: A hard x-ray nanoprobe beamline at MAX IV; SPIE Optical Engineering+ Applications, 2013; International Society for Optics and Photonics: 2013; pp 88510L-88510L-10.

(15) Döring, F.; Robisch, A. L.; Eberl, C.; Osterhoff, M.; Ruhlandt, A.; Liese, T.; Schlenkrich, F.; Hoffmann, S.; Bartels, M.; Salditt, T.; Krebs, H. U. Opt. Express 2013, 21 (16), 19311-19323.

(16) Mimura, H.; Handa, S.; Kimura, T.; Yumoto, H.; Yamakawa, D.; Yokoyama, H.; Matsuyama, S.; Inagaki, K.; Yamamura, K.; Sano, Y.; Tamasaku, K.; Nishino, Y.; Yabashi, M.; Ishikawa, T.; Yamauchi, K. Nat. Phys. 2010, 6 (2), 122-125.

(17) Segura-Ruiz, J.; Martínez-Criado, G.; Chu, M. H.; Geburt, S.; Ronning, C. Nano Lett. 2011, 11 (12), 5322-5326.

(18) del Alamo, J. A. Nature 2011, 479, 317.

(19) Taraci, J. L.; Hÿtch, M. J.; Clement, T.; Peralta, P.; McCartney, M. R.; Drucker, J.; Picraux, S. T. Nanotechnology 2005, 16 (10), 2365.

(20) Johansson, J.; Dick, K. A. CrustEngComm 2011, 13 (24), 71757184.

(21) Bryllert, T.; Wernersson, L. E.; Froberg, L. E.; Samuelson, L. IEEE Electron Device Lett. 2006, 27 (5), 323-325.

(22) Tomioka, K.; Yoshimura, M.; Fukui, T. Nature 2012, 488, 189.

(23) Riel, H.; Wernersson, L.-E.; Hong, M.; del Alamo, J. A. MRS Bull. 2014, 39 (8), 668-677.

(24) Wallentin, J.; Anttu, N.; Asoli, D.; Huffman, M.; Åberg, I.; Magnusson, M. H.; Siefer, G.; Fuss-Kailuweit, P.; Dimroth, F.; Witzigmann, B.; Xu, H. Q.; Samuelson, L.; Deppert, K.; Borgström, M. T. Science 2013, 339 (6123), 1057-1060.

(25) Cui, Y.; Duan, X. F.; Hu, J. T.; Lieber, C. M. I. Phvs. Chem. B 2000, 104 (22), 5213-5216.

(26) Willander, M.; Nur, O.; Zhao, Q. X.; Yang, L. L.; Lorenz, M.; Cao, B. Q.; Pérez, J. Z.; Czekalla, C.; Zimmermann, G.; Grundmann, M.; Bakin, A.; Behrends, A.; Al-Suleiman, M.; El-Shaer, A.; Mofor, A. C.; Postels, B.; Waag, A.; Boukos, N.; Travlos, A.; Kwack, H. S.; Guinard, J.; Dang, D. L. S. Nanotechnology 2009, 20 (33), 332001.

(27) Waleed, A.; Tavakoli, M. M.; Gu, L.; Wang, Z.; Zhang, D.; Manikandan, A.; Zhang, Q.; Zhang, R.; Chueh, Y.-L.; Fan, Z. Nano Lett. 2017, 17 (1), 523-530.

(28) Molassioti, A.; Scholz, F.; Gao, Y. L. Crust. Growth 1990, 102 (4), 974-978.
(29) Otnes, G.; Heurlin, M.; Graczyk, M.; Wallentin, J.; Jacobsson, D.; Berg, A.; Maximov, I.; Borgström, M. T. Nano Res. 2016, 9 (10), $2852-2861$.

(30) Martinez-Criado, G.; Villanova, J.; Tucoulou, R.; Salomon, D.; Suuronen, J. P.; Laboure, S.; Guilloud, C.; Valls, V.; Barrett, R.; Gagliardini, E.; Dabin, Y.; Baker, R.; Bohic, S.; Cohen, C.; Morse, I. I. Sunchrotron Radiat. 2016, 23, 344-352.

(31) Berg, A.; Lenrick, F.; Vainorius, N.; Beech, J. P.; Wallenberg, L. R.; Borgström, M. T. Nanotechnology 2015, 26 (43), 435601.

(32) Kim, Y.; Joyce, H. J.; Gao, Q.; Tan, H. H.; Jagadish, C.; Paladugu, M.; Zou, J.; Suvorova, A. A. Nano Lett. 2006, 6 (4), 599604.

(33) Berg, A.; Caroff, P.; Shahid, N.; Lockrey, M. N.; Yuan, X.; Borgström, M. T.; Tan, H. H.; Jagadish, C. Nano Res. 2017, 10 (2), $672-682$.

(34) Wallentin, J.; Messing, M. E.; Trygg, E.; Samuelson, L.; Deppert, K.; Borgström, M. T. I. Cryst. Growth 2011, 331 (1), 8-14.

(35) Ameruddin, A. S.; Caroff, P.; Tan, H. H.; Jagadish, C.; Dubrovskii, V. G. Nanoscale 2015, 7 (39), 16266-16272.

(36) Otnes, G.; Heurlin, M.; Zeng, X.; Borgström, M. T. Nano Lett. 2017, 17 (2), 702-707.

(37) Chu, M. H.; Martínez-Criado, G.; Segura-Ruiz, J.; Geburt, S.; Ronning, C. Phys. Status Solidi A 2014, 211 (2), 483-487.

(38) Belter, M.; Sajnóg, A.; Barałkiewicz, D. Talanta 2014, 129, 606-616.

(39) Currie, L. A. Anal. Chim. Acta 1999, 391 (2), 127-134.

(40) Currie, L. A. Nomenclature in evaluation of analytical methods, including detection and quantification capabilities (IUPAC Recommendations 1995). Pure Appl. Chem. 1995, 67, 1699-1723.

(41) Goto, H.; Nosaki, K.; Tomioka, K.; Hara, S.; Hiruma, K.; Motohisa, J.; Fukui, T. Appl. Phys. Express 2009, 2 (3), 035004.

(42) Li, N.; Tan, T. Y.; Gosele, U. Appl. Phys. A: Mater. Sci. Process. 2008, 90 (4), 591-596.

(43) Hjort, M.; Wallentin, J.; Timm, R.; Zakharov, A. A.; Andersen, J. N.; Samuelson, L.; Borgström, M. T.; Mikkelsen, A. Appl. Phvs. Lett. 2011, 99 (23), 233113.

(44) Heurlin, M.; Anttu, N.; Camus, C.; Samuelson, L.; Borgström, M. T. Nano Lett. 2015, 15 (5), 3597-3602.

(45) Zeng, X.; Otnes, G.; Heurlin, M.; Mourão, R. T.; Borgström, M. T. Nano Res. 2018, 11, 2523.

(46) Casadei, A.; Krogstrup, P.; Heiss, M.; Röhr, J. A.; Colombo, C.; Ruelle, T.; Upadhyay, S.; Sørensen, C. B.; Nygård, J.; Fontcuberta i Morral, A. Appl. Phys. Lett. 2013, 102 (1), 013117.

(47) Kirkham, R.; Dunn, P. A.; Kuczewski, A. J.; Siddons, D. P.; Dodanwela, R.; Moorhead, G. F.; Ryan, C. G.; Geronimo, G. D.; Beuttenmuller, R.; Pinelli, D.; Pfeffer, M.; Davey, P.; Jensen, M.; Paterson, D. J.; Jonge, M. D. d.; Howard, D. L.; Küsel, M.; McKinlay, J. AIP Conf. Proc. 2010, 1234 (1), 240-243.

(48) Eriksson, M.; van der Veen, J. F.; Quitmann, C. I. Sunchrotron Radiat. 2014, 21 (5), 837-842.

(49) Xie, P.; Hu, Y. J.; Fang, Y.; Huang, J. L.; Lieber, C. M. Proc. Natl. Acad. Sci. U. S. A. 2009, 106 (36), 15254-15258.

(50) Borgström, M. T.; Wallentin, J.; Trägårdh, J.; Ramvall, P.; Ek, M.; Wallenberg, L. R.; Samuelson, L.; Deppert, K. Nano Res. 2010, 3 (4), 264-270.

(51) Jacobsson, D.; Persson, J. M.; Kriegner, D.; Etzelstorfer, T.; Wallentin, J.; Wagner, J. B.; Stangl, J.; Samuelson, L.; Deppert, K.; Borgström, M. T. Nanotechnology 2012, 23 (24), 245601.

(52) Solé, V. A.; Papillon, E.; Cotte, M.; Walter, P.; Susini, I. Spectrochim. Acta, Part B 2007, 62 (1), 63-68. 